\title{
Comparison of postoperation bone defects healing of alveolar processes of maxilla and mandible with the use of Bio-Gen and Bio-Oss.
}

\author{
Joanna Śmieszek-Wilczewska ${ }^{1}$, Rafał Koszowski ${ }^{2}$, Jacek Pająk ${ }^{3}$ \\ ${ }^{1} \mathrm{PhD}$, Chair and Department of Oral Surgery in Bytom Silesian Medical University, Poland. \\ ${ }^{2}$ Professor, Chair and Department of Oral Surgery in Bytom Silesian Medical University, Poland . \\ ${ }^{3} \mathrm{PhD}$, Chair of Morphology and Department of Histopathology Silesian Medical University.
}

Correspondence:

Dr Joanna Śmieszek-Wilczewska

Pl.Akademicki 17, 41-902 Bytom

E-mail: tallen@poczta.onet.pl

Received: 27/12/2009

Accepted: 15/03/2010

\author{
Śmieszek-Wilczewska J, Koszowski R, Pająk J. Comparison of postope- \\ ration bone defects healing of alveolar processes of maxilla and mandible \\ with the use of Bio-Gen and Bio-Oss.. J Clin Exp Dent. 2010;2(2):e60-6. \\ http://www.medicinaoral.com/odo/volumenes/v2i2/jcedv2i2p60.pdf

\begin{abstract}
Bone defects of mandible and maxilla alveolar processes have often iatrogenic origins, because they appear as a consequence of performed operations. Bone atrophy may be stopped by filling bone defects with augmentative biomaterial directly after an oral surgery procedure. To achieve this aim augmentative biomaterials are used. Many years of experimental studies confirmed xenogenic biomaterials as the most effective.

The aim of this study was clinical assessment of bone lesions healing with the use of Bio-Gen and Bio-Oss materials. A comparison of radiological imaging of healed defects was performed, as well as histological analysis of biopsies obtained from the defect sites. The structure analysis of both materials was performed with the use of electron microscopy. Chemical ingredients of analyzed biomaterials were assessed with the use of X-ray microanalysis. Postoperation follow-up in all groups was done. Histological and radiological analysis of regeneration course were performed. Radiological analysis was performed with the use of Digora 2,1 system, which allows to asses optical density of images. Histopathologic regenerates were analyzed morfometrically.

The results of this study confirmed effectiveness of Bio-Gen and Bio-Oss materials in regeneration of bone defects. Six month long follow-up period confirmed a significant increase of the optical density of regenerates augmented with Bio-Gen and Bio-Oss comparing to lesions healed without biomaterial augmentation.
\end{abstract}

Key words: Biomaterials, bone defects, guided bone regeneration. 


\section{Introduction}

Surgical procedures carried out in the area of alveolar processes of maxilla and the alveolar processes of mandible lead to an irreversible loss of the bone tissue. Bone losses caused during the extraction of the unerupted teeth, tooth roots left over, enucleation of cysts and odontogenic tumours often make subsequent prosthetic treatment with the use of conventional prosthesis more difficult, and the application of implants - impossible $(1,2)$. Remote, reconstructive augmentative procedures aiming at the reconstruction of the lost bone base are complicated, marked with a high risk of failure and they often do not bring satisfactory results. Therefore, the chosen course of action should consist in completing the losses right after their creation (3). At present the most commonly used method to achieve it is the guided bone regeneration, using bone-replacing materials and/ or autogenic bone transplants, as well as resorbable and non-resorbable barrier membranes.

Biomaterials, constituting - together with the autogenic tissue - a constituent of the augmentation material, or independently completing the lost tissues, are used more and more often to complete bone losses. Among the biomaterials used in the augmentative procedures a special position is occupied by animal-derivative preparations, as they have a structure similar to the human bone. It is one of the factors which influences the acceleration of osteoconduction, thanks to which these materials have an advantage over synthetic materials. They are characterized by osteotrophism and the ability of osteointegration. They contain the biological apatite of bones, with fewer hydroxyl groups and a larger number of carbonic ions than in synthetic materials. In this group there are the preparations Bio-Oss and Bio-Gen (4-6).

The Bio-Oss material (Biomaterials Geistlich, Switzerland) is produced out of totally deproteinized bovine bones, it has a porous internal surface and a crystalline structure (7). It causes osteoconductive action, and its porous surface is subject to vascularisation and permeation with collagen fibres, which leads to osteogenesis. It resorbs itself slowly, being integrated in the process of natural remodelling $(8,9)$. The ratio of the calcium ions to phosphorus is $2: 1$. The size of the crystals of the Bio-Oss material is $10-60 \mathrm{~nm}$, whereas in the human bone their size is $50-90 \mathrm{~nm}$. The total porousness of the Bio-Oss preparation equals $60 \%$, similarly to the human bone $(10,11)$.

It is subject to strong chemical processes, consisting in submerging the materials in strong bases $(\mathrm{pH} \mathrm{13)}$ for over 4 hours. Sterilization of the Bio-Oss material takes place with the application of the Gamma rays (12). In the production of the preparation, bovine bones are used, coming from selected animal breeding. The material comes from the limbs of the animals. Calf bones, unlike their brain, viscera or spinal cord, belong to tissues free from prions as even in case of infected animals no contagious material has been found in bones (13). The process of the preparation production is controlled by a Quality Control System, verified every year by independent experts and international commissions. Consequently, the Bio-Oss preparation has been qualified as a safe material, not causing any danger of transmitting prions of the bovine spongiform encephalopathy disease (13).

The Bio-Gen preparation (Bioteck, Italy) is a material coming from the horse bone deantigenized in the temperature of $125^{\circ} \mathrm{C}$. It is hydrophilic, in contrast to the hydrophobic Bio-Oss material. Bio-Gen, thanks to its spatial structure, gives a possibility of migration and growth of capillaries. The size of the crystals of the preparation oscillates between 15 and $60 \mathrm{~nm}$. Sterilization of the Bio-Gen material takes place with the application of ion Beta radiation in the dose of $25 \mathrm{KgJ}$. Bio-Gen is a non-calcifying material, produced in the chemical / enzymatic process in a low temperature, which allows to eliminate organic parts in such a way that the structure of the bone trabeculas is not subject to any change. The Bio-Oss material, in contrast to the Bio-Gen material, is subject to the calcification process (14). Both preparations have been tested and accepted to be used on patients in the European Union and in the USA.

The aim of this study was to compare the structure of xenogenic materials Bio-Gen and Bio-Oss. Clinically evaluate healing of bone wounds after the application of the preparations Bio-Gen and Bio-Oss. Compare the radiological image of the bone regeneration in post-surgical losses of alveolar processes of maxilla and mandible after the application of the preparations Bio-Gen and Bio-Oss, and also compare histopathologic images of the regenerate created in the post-surgical bone losses after the application of the preparations Bio-Gen and Bio-Oss.

\section{Material and methods}

In order to evaluate the surface structure, the size of the particles, the contents of elements and the differences between both materials, a test in the JCXA 733 X-ray analyzer by JOEL (Japan) was performed, with the use of secondary electrons. Magnification 40-6000x was applied.

Clinical research was conducted on a group of 90 patients (57 women and 33 men) treated in the consulting centre of the Chair and Department of Oral Surgery in Bytom of the Silesian Medical University. It comprised persons aged over 18, without any systemic illnesses. Pregnant women were excluded from the research. Nonselectively chosen patients with bone defects in the area of dental processes of the jaw and the alveolar part of the mandible were qualified for the procedure. The defects had appeared as a result of the apicectomy combined with enucleation of root cysts or periapical granulomas, 
as well as teeth extraction prior to the planned implantations. All procedures were conducted on the frontal section of the alveolar process of maxilla or the alveolar part of the mandible and in the area of premolar teeth in order to obtain the possibility to observe the process of healing of the bone defects of a similar structure type. The procedures were conducted in the condition of an operating room with the use of a local anesthesia. The bone defects were filled up with the Bio-Gen (Osteoplant Mix) and Bio-Oss (Bio-Oss Spongiosa) preparations.

In case of 30 patients the bone defects were filled up with the Bio-Gen preparation of the particle dimensions of $0.5-1 \mathrm{~mm}$ (1 st examined group), and in second group (30 patients) the bone defects were filled up with BioOss material of the particle dimensions of $0,5-1 \mathrm{~mm}$. In the control group of 30 persons post-operative bone defects were left for self-healing on the basis of the forming clot. The choice between the biomaterial application or leaving the bone loss for self-healing was made at random in order to ensure the research randomization. After the procedure, the antibiotic cover was recommended, in the form of synthetic penicillin's derivatives, and in case of an allergy to these preparation - clindamycin, as well as rinsing the oral cavity with $0.12 \%$ chlorhexidine gluconate. The sutures were removed after 7 days.

In the clinical evaluation, the attention was drawn to the presence of the post-operative oedema of soft tissues, haematomas, the wound dehiscence, the time after which the healing was completed and the configuration of the alveolar process after the healing of the wound (colouring of the mucous membrane, shape of the alveolar process - collapse, bulge). Six months after the procedure another evaluation was carried out, taking into consideration the presence of fistulas, the tissue colouring, the presence of scars and the configuration of the alveolar processin the place of the surgical procedure (Table 1).

In order to radiologically evaluate the process of the bone regeneration, intraoral dental radiograms were made directly after the surgical procedure (initial radiogram) and after 6 months (check-up radiogram). The technique of the right angle was used. In order to standardize the projection conditions, the handle fixing the film was fixed inside the oral cavity with the use of silicone mass Regidur produced by Bisico.

The radiograms obtained were used digital technique. All radiograms were entered to the Digora 2.1 software which was used to perform a statistical analysis according to its own methodology. The Digora 2.1 software enables an objective evaluation of X-ray photographs thanks to a digital radiodensitometric analysis. After making the check-up radiograms (after the period of 6 months), the numerical values of density were compared and they were depicted in a graphical form with the measurements of density of the initial radiograms. The numerical data obtained in this manner were entered into a calculation sheet and subject to a statistical analysis. Firstly, in the initial X-ray image the line dimensions (length and width) of the lesion were determined, indicated as the examined area. Next, accessory lines limiting a four-sided field (examined field) were laid out, comprising the examined area. The accessory lines were laid out in such a way so as to make them tangent to the examined area. After their intersection they should limit the field with the closest surface to the surface of the examined area. This field is called an examined field. At this stage a measurement of the density of the examined field was made in a form of a numerical maximum (Gmax), minimum (Gmin) and average (Gm) value and its presentation in a graphical form as a

\begin{tabular}{|c|c|c|c|c|c|c|}
\hline & $\begin{array}{l}\text { CONTROL } \\
\text { GROUP }\end{array}$ & GROUP I & GROUP II & VALUE $p$ \\
\hline \multirow{3}{*}{\multicolumn{2}{|c|}{$\begin{array}{l}\text { Swelling in I day after surgical } \\
\text { procedure }\end{array}$}} & 0 & 6 & 9 & 11 & \multirow{3}{*}{0,402} \\
\hline & & + & 18 & 15 & 17 & \\
\hline & & ++ & 6 & 6 & 2 & \\
\hline \multirow{2}{*}{\multicolumn{2}{|c|}{$\begin{array}{c}\text { Swelling in } 7 \text { day after surgical } \\
\text { procedure }\end{array}$}} & 0 & 27 & 28 & 28 & \multirow{2}{*}{0,856} \\
\hline & & + & 3 & 2 & 2 & \\
\hline \multirow{2}{*}{\multicolumn{2}{|c|}{$\begin{array}{l}\text { Hematoma in } 1 \text { day after surgi- } \\
\text { cal procedure }\end{array}$}} & 0 & 22 & 22 & 21 & \multirow{2}{*}{0,946} \\
\hline & & + & 8 & 8 & 9 & \\
\hline \multirow{2}{*}{\multicolumn{2}{|c|}{$\begin{array}{l}\text { Hematoma in } 7 \text { day after surgi- } \\
\text { cal procedure }\end{array}$}} & 0 & 29 & 30 & 30 & \multirow{2}{*}{0,364} \\
\hline & & + & 1 & 0 & 0 & \\
\hline \multirow{2}{*}{$\begin{array}{l}\text { Healing of } \\
\text { the wound }\end{array}$} & \multicolumn{2}{|c|}{ primary adhesion } & 23 & 26 & 25 & \multirow{2}{*}{0,587} \\
\hline & \multicolumn{2}{|c|}{ growth of granulation } & 7 & 4 & 5 & \\
\hline \multirow{3}{*}{$\begin{array}{l}\text { Configuration } \\
\text { of the alveo- } \\
\text { lar process }\end{array}$} & \multicolumn{2}{|c|}{ collapse } & 10 & 1 & 0 & $<0.001$ \\
\hline & \multicolumn{2}{|l|}{ bulge } & 0 & 9 & 6 & $<0.001$ \\
\hline & \multicolumn{2}{|l|}{ regular } & 20 & 20 & 24 & 0.329 \\
\hline
\end{tabular}

Table 1. Statistical date of surgical procedure 
histogram and the field density profile. It allowed to determine the initial parameters of the radiological image, which constituted a reference for the values obtained as a result of measurements on the follow-up X-ray images made after 6 month.

The statistical analysis was performed with the use of the software Statistica 7.1 Pl. The values calculated for measureable variables were presented as an arithmetic mean with a standard deviation (SD) or standard error of mean (SEM). The normality of the decomposition was tested with the use of Shapiro-Wilk's test, and the variance homogeneity - with the use of Leven's test. The comparative analysis for more than two groups was performed using the analysis of variances, the ANOVA test of ranges of Kruskal-Wallis and post-hoc tests (Newman-Keulus's test or test for multiple comparisons). For the comparison of two groups a test $t$, a test $t$ with an independent estimation of variances or test $U$ of MannWhitney were used. For connected variables the analysis was performed with the use of the $T$ test for dependant trials or with the use of Wilcoxon's test. The Chi-square statistics was used for the evaluation of qualitative variables. Changes at the level of significance $p<0.05$ were assumed to be statistically interchangeable.

In case of 10 patients where post-extraction alveoli were filled with biomaterial in order to make implantation possible, a histopathologic test of the regenerate was performed. After 6 month, during drilling the bed for an implant, material was taken for histopathologic evaluation with the use of a miniaturized trephine with the diameter of $2 \mathrm{~mm}$. The trephine was used prior to the use of a pilot drill, which did not cause the patient to suffer a loss of the new tissue without any need. The preparations obtained were subject to colouring with the use of the hematoxylin - eosine method (HE). The histopathologic preparations evaluated in a light microscope Olympus AX70 with the enlargement of $125-320$ times. In order to were objectify the research, a morphometric analysis of morphometric images was performed. The morphometric analysis was performed after taking pictures with the enlargement of $10 \times 10$ with the application of the Motic software.

\section{Results}

The analysis in the electron microscope did not exhibit any significant differences in the structure of the BioGen and Bio-Oss preparations. The average size of particles of both preparations did not exceed $1 \mathrm{~mm}$. The shape of the particles was irregular, whereas the particles of the Bio-Oss preparation had rounder shapes, including narrowings. The particles of the Bio-Gen preparation, on the other hand, were more regular, with sharp edges, close to a straight line. It may result from different hardness of both materials. The tests also exhibited greater porosity (numerous micropores and directed microfissu- res) of the Bio-Gen preparation. In the Bio-Oss material, smooth fractures of particles area were confirmed, with compact concentration of the bone material, with few microfissures. The chemical composition of both materials was very similar. Only higher contents of oxygen, sodium and magnesium were confirmed in Bio-Gen preparation.

Due to the fact that in the control group the bone defects were left for self-healing, the value of Gmin, Gmax and Gmean was lower than in the examined group, where these defects were filled up with biomaterial, visible as a shading in the radiological image. The statistical significance of the difference of the Gmin value between the examined group and the control group directly after the procedure was confirmed $(p<0.001)$. A significant statistical difference was also observed for the value of Gmax $(p<0.001)$ and for the value of Gmean $(p<0.001)$. In the 6-month-long observation period the statistical analysis confirmed a significant difference in the increase of the Gmin value between the first and second examined group and the control group ( $<<0.001)$. A statistically significant increase of the Gmax value in 6 months after the procedure was also confirmed between the examined group and the control one $(\mathrm{p}<0.001)$. A statistical significance in the increases of Gmean in the examined group with reference to the control group was confirmed, as well (I, II $\mathrm{p}<0.001$ ).

In the first examined group (Bio-Gen preparation) 6 months after the procedure, an increase of the Gmin value was observed, from 77.47 to $100.10(p<0.001)$, Gmax from 148.90 to $179.43(\mathrm{p}<0.001)$ and Gmean from 107.97 to $135.80(p<0.001)$. Statistical calculations showed a statistically significant increase of the optical density of a radiological image during the 6-month-long observation period.

In the second examined group (Bio-Oss material) 6 months after the procedure, an increas of the Gmin value was observed, from 70,90 to 82.23 ( $\mathrm{p}=0.001$ ), Gmax from 156.73 to $174.13(\mathrm{p}=0.02)$ and Gmean from 106.05 to $123.76(\mathrm{p}<0.001)$. Statistical calculations showed a statistically significant increase of the optical $G$ mean density of a radiological image during the 6-month-long observation period.

In the control group after 6 months from the surgical procedure, the Gmin value increased from 34.90 to 42.43 ( $p=0.013)$, Gmax from 97.40 to $112.22(p<0.001)$, and Gmean from 61.00 to $73.95(\mathrm{p}<0.001)$. The statistical significance in the control group was observed for Gmin, Gmax and Gmean (Fig. 1-3).

Statistical significance was observed in the increase of Gmean between the 1st and 2nd examined group $\mathrm{p}=0.028$. Statistical significance was also confirmed in case of increases of Gmean in both groups when compared to he control group.

In case of 10 patients, where after the extraction of teeth 


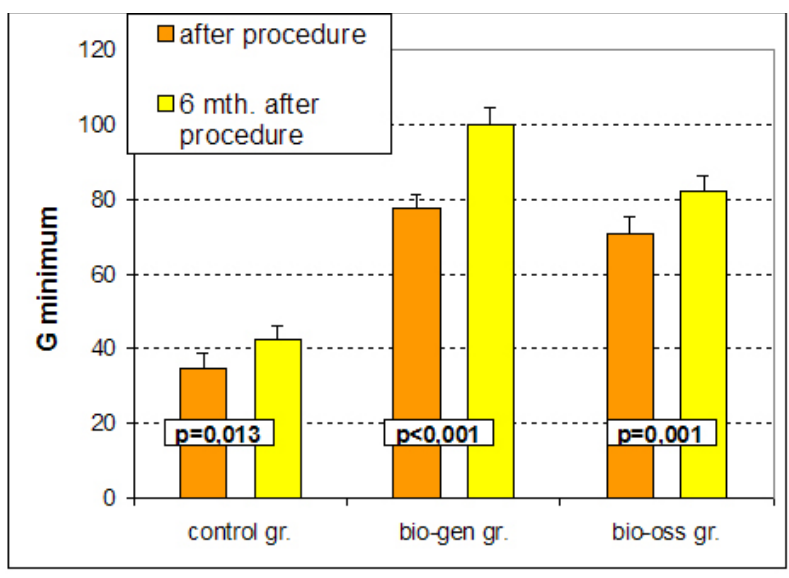

Fig.1. Increase of the Gmin value in I and II examined groups and in the control group during 6 month-long observation period.

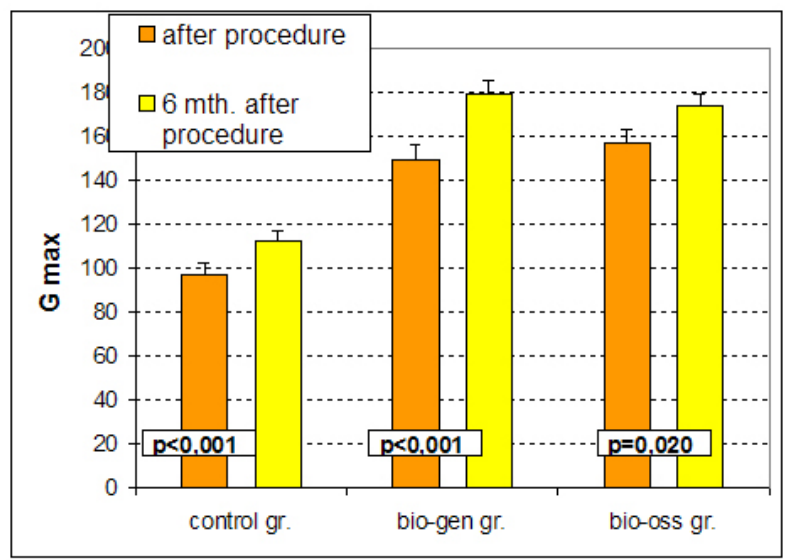

Fig. 2. Increase of the Gmax value in I and II examined groups and in the control group during 6 month-long observation period.

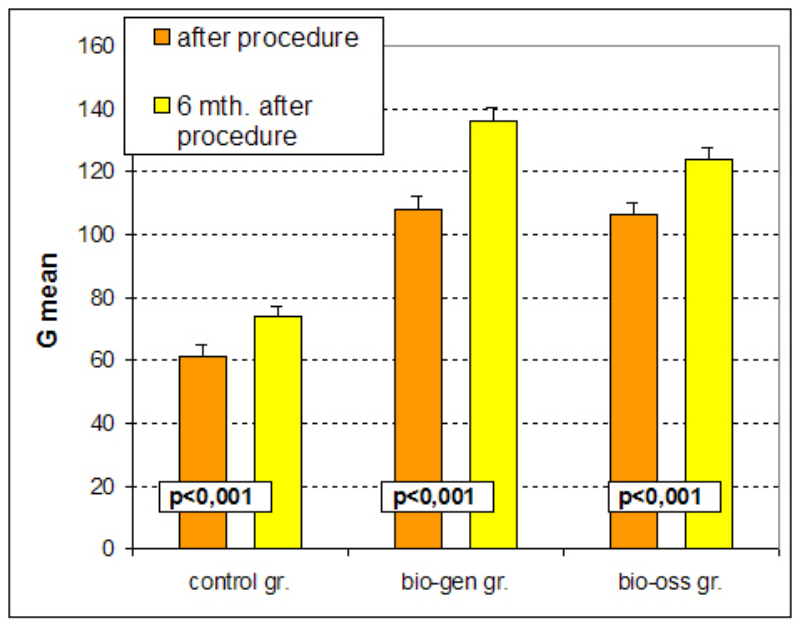

Fig. 3. Increase of the Gmean value in I and II examined groups and in the control group during 6 month-long observation period.

the dental alveoli were filled with the biomaterial, after 6 month the augmentation areas were revealed in order to introduce dental implants. After the incision of tissues and the preparation of the mucous membrane and periosteum it was stated that the alveoli where the Bio-Gen
-1 st examined group ( 5 persons) and the Bio-Oss -2 nd examined group ( 5 persons) preparations had been introduced, were filled with a solid tissue, macroscopically only slightly different from the bone surrounding it.

The histopathologic examination of the regenerate formed after the introduction of the Bio-Gen preparation (1st examined group) proved the presence of the connective fibrous tissue without any inflammatory infiltrations and absorption foci. Only single inactive lymphocytes were present. Between the fibres of the connective tissue there were amorphous acidophilic masses, corresponding to the unresorbed biomaterial fragments and regular mature bone trabeculae, without stimulated osteoblasts. There were no osteoclasts.

The histopathologic examination of the regenerate formed after the application of the Bio-Oss preparation (2nd group) proved normally calcified bone trabeculae with osteocytes. In the spaces between the trabeculae there were young not fully formed bone trabeculae and the connective fibrous tissue. In the connective tissue and in some spaces between the trabeculae minor unresorbed fragments of the biomaterial were discovered (amorphous acidophilic masses). There were no inflammatory infiltrations.

\section{Discussion}

The bone defects of alveolar processes of maxilla and the alveolar part of the mandible are often of an iatrogenic character as they are a consequence of surgical procedures. Cawood and Howell (15) pointed out that after a tooth extraction or a cystectomy accompanying the apicectomy, a progressive atrophy of the atrial lamina is observed, leading to narrowing the dental process in the place of the previous surgical procedure. This opinion was confirmed with the research carried out by Nevins et al. (16), who assessed the width of the dental process on cross sections of the computer tomography directly after the procedure, and then after 30 and 90 days from the date of the tooth extraction. They affirmed that the bone defect progressing from the atrial surface in the direction of the proper oral cavity can be stopped by filling up the post-operative defect with an augmentative material, directly after its appearance. The report is also confirmed with this research. In the control group where the bone defects were left for self-healing, sinking in of the atrial surface of the dental process was much more common than in the case of defects subjected to augmentation. With the development of implantology the problem of augmentative procedures has assumed a new dimension. It has forced the researchers to search for methods of the regeneration of bone defects, enabling to avoid complicated regeneration procedures, often using autogenic grafts taken from distance donor locations outside the oral cavity. At present there are numerous bone-replacing materials available. An ideal preparation should be 
characterized with bioconsistency and possess osteoconductive and osteoinductive properties (17). Long-term experience proved the best effectiveness of xenogenic preparations, made of animal bone. Both their structure close to the human bone, and the resorption time allow for osteogenesis in the system of pores in these biomaterials and a gradual replacement with the recipient's bone (18). It enables to generate an appropriate volume of a valuable bone tissue, fulfilling the conditions of a bed for dental implants (19). Decalcified bovine bone, subject to osteointegration with the newly-generated bone tissue, proved to be highly biocompatible and osteoinductive. The author's research exhibited in a 6-month-long observation period a decisively significantly greater increase of the optical density of the bone losses augmented with both biomaterials used in comparison to the bone wounds left to be healed by themselves. At the same time, a slight, but statistically significantly greater increase of the optical density in the losses completed with the Bio-Gen preparation was observed. These data may confirm a faster course of bone generation after the application of this material. The author's histopathologic tests performed after 6 months from the augmentation confirmed the presence of unresorbed particles of both biomaterials, surrounded by the connective tissue and the newly generated bone tissue. However, as the group subject to morphometric analysis was not numerous enough it is impossible to draw clear-cut conclusions relating to the speed of their resorption and bone modeling, indicating the difference in the bone regeneration course after the application of the Bio-Gen and Bio-Oss preparations.

The author's research confirmed a high quality of both evaluated preparations and the possibility to reach the desired clinical effects after applying them. The radiological evaluation of the bone regeneration indicated its slightly quicker course in case of the augmentation with the use of the Bio-Gen preparation. This can be connected with greater porosity of the biomaterial, rendering angiogenesis, and subsequently bone modeling, easier. Clergeau et al. (20) on the basis of experimental research emphasized the significance of porosity of bone-substituting preparations deriving from deproteinized animal bone. The histopathologic evaluation of the regenerates confirmed comparative results in case of applying both biomaterials. In future the research continuation shall enable to perform a detailed statistical analysis of morphometric measurements results on the basis of a larger number of microscopic preparations. It is planned to obtain tissue material also within a longer observation period than 6 months, which will enable a detailed investigation of the resorption process for both materials. Results of this study confirmed effectiveness of Bio-Gen and Bio-Oss materials in regeneration of bone defects. Six month follow-up period confirmed significant in- crease of optical density of regenerates augmented with Bio-Gen and Bio-Oss comparing to lesions healed without biomaterial augmentation. Bio-Gen comparing to Bio-Oss was more effective in optical density increasing. Regenerates assessed histologically were similar in Bio-Gen and Bio-Oss group.

\section{References}

1. O'Brien TP, Hinrichs JE, Schaffer EM. The prevention of localized ridge deformities using guided tissue regeneration. J Periodontol. 1994; 65: 17-24.

2. von Arx T, Buser D. Horizontal ridge augmentation using autogenous block grafts and the guided bone regeneration technique with collagen membranes: a clinical study with 42 patients. Clin Oral Implants Res. 2006; 17: 359-66.

3. Goldberg VM, Stevenson S. Natural history of autografts and allografts. Clin Orthop Relat Res. 1987; 225: 7-16.

4. McAllister BS, Margolin MD, Cogan AG, Taylor M, Wollins J. Residual lateral wall defects following sinus grafting with recombinant human osteogenic protein-1 or Bio-Oss in the chimpanzee. Int J Periodontics Restorative Dent. 1998;18:227-39.

5. Simion M, Dahlin C, Trisi P, Piattelli A. Qualitative and quantitative comparative study on different filling materials used in bone tissue regeneration: a controlled clinical study. Int J Periodontics Restorative Dent. 1994; 14: 199- 215.

6. Wetzel AC, Stich H, Caffesse RG. Bone apposition onto oral implants in the sinus area filled with different grafting materials. A histological study in beagle dogs. Clin Oral Implants Res. 1995;6:155-63. 7. Açil Y, Terheyden H, Dunsche A, Fleiner B, Jepsen S. Three-dimensional cultivation of human osteoblast-like cells on highly porous natural bone mineral. J Biomed Mater Res. 2000;51:703-10.

8. Camelo M, Nevins ML, Schenk RK, Simion M, Rasperini G, Lynch SE, Nevins M. Clinical, radiographic, and histologic evaluation of human periodontal defects treated with Bio-Oss and Bio-Gide. Int J Periodontics Restorative Dent. 1998;18:321-31.

9. Schlegel AK, Donath K. BIO-OSS---a resorbable bone substitute? J Long Term Eff Med Implants. 1998;8:201-9.

10. Benke D, Olah A, Möhler H. Protein-chemical analysis of Bio-Oss bone substitute and evidence on its carbonate content. Biomaterials. 2001;22:1005-12.

11. Carmagnola D, Berglundh T, Araújo M, Albrektsson T, Lindhe J. Bone healing around implants placed in a jaw defect augmented with Bio-Oss. An experimental study in dogs. J Clin Periodontol. 2000; 27: 799-805.

12. Araújo MG, Carmagnola D, Berglundh T, Thilander B, Lindhe J. Orthodontic movement in bone defects augmented with Bio-Oss. An experimental study in dogs. J Clin Periodontol. 2001; 28: 73-80.

13. Wenz B, Oesch O, Horst M. Analysis of the risk of transmitting bovine spongiform encephalopathy through bone grafts derived from bovine bone. Biomaterials. 2001; 22: 1599-606.

14. Bianchi SD, Lojacono A, Nevins M, Ramieri G, Corrente G, Martuscelli $\mathrm{G}$, et al. The use of replicate resin models in the treatment of maxillary sinus augmentation patients. Int J Periodontics Restorative Dent. 1997; 17:348-57.

15. Cawood JI, Howell RA. A classification of the edentulous jaws. Int J Oral Maxillofac Surg. 1988; 17: 232-6.

16. Nevins M, Camelo M, De Paoli S, Friedland B, Schenk RK, Parma-Benefati S, et al. A study of the fate of the buccal wall of extraction sockets of teeth with prominent roots. Int J Periodontics Restorative Dent. 2006; 26:19-29.

17. Shanaman RH. A retrospective study of 237 sites treated consecutively with guided tissue regeneration. Int J Periodontics Restorative Dent. 1994; 14: 293-301.

18. Camelo M, Nevins ML, Schenk RK, Simion M, Rasperini G, Lynch SE, Nevins M. Clinical, radiographic and histologic evaluation of human periodontal defects treated with Bio-Oss and Bio-Gide. Int J Periodontics Restorative Dent. 1998; 18: 321-31. 
19. Zitzmann NU, Schärer P, Marinello CP. Long-term results of implants treated with guided bone regeneration: a 5- year prospective study. Int J Oral Maxillofac Implants. 2001; 16: 355-66.

20. Clergeau LP, Danan M, Clergeau-Guérithault S, Brion M. Healing response to anorganic bone implantation in periodontal intrabony defects in dogs. Part I. Bone regeneration. A microradiographic study. J Periodontol. 1996; 67: 140-9. 